\title{
CORRECTIONS
}

\section{Diagnosis and management of cellulitis}

Some corrections and clarifications need to be made to this Clinical Review (BMJ 2012;345:e4955, doi:10.1136/bmj.e4955). In Table 1, "Treatment recommendations for cellulitis based on organisms," the antibiotic ciprofloxacin is incorrectly spelt as "ciprofloxacillin." "Ciprofloxacillin" is listed three times as the antibiotic choice for the clinical presentations of: "Freshwater exposure," "Necrotising fasciitis," and "Butchers and fish handlers." In all three instances the antibiotic should have been spelt as ciprofloxacin. In Table 1 it should also be noted that the organism "Erysipelothrix" should be treated with penicillin and that ciprofloxacin (which the table recommends) should be prescribed if the patient is allergic to penicillin.

Additionally, in Table 1 the organism "Clostridium perfringens" is recorded as the causative agent for "Necrotising fasciitis," when it should have been more accurately summarised as polymicrobial; with common causes including group A streptococci, Staphylococcus aureus, and anaerobes.

Lastly, under the "What is the treatment of cellulitis?" section, the first line of the third paragraph states that the "CREST guidelines still recommend amoxicillin or flucloxacillin for the majority of cases of cellulitis caused by S aureus, Streptococcus, or when the organism has not been identified." In fact, the CREST guidelines do not recommend amoxicillin and this sentence should have cited only flucloxacillin.

Cite this as: BMJ 2012;345:e5553

๑ B BMJ Publishing Group Ltd 2012 